\title{
Cosmic Equation of state from Strong Gravitational Lensing Systems
}

\author{
Beata Malec \\ Copernicus Center for Interdisciplinary Studies, Cracow, Poland \\ E-mail: beata.malec@us.edu.pl
}

\section{Marek Biesiada}

University of Silesia, Katowice, Poland

E-mail: marek.biesiada@us.edu.pl

Here we present the joint analysis of five dark energy models using the data from Type Ia Supernovae (data from recent Union2 compilation), Cosmic Microwave Background (CMB) shift parameter R (WMAP7 data), Baryon Acoustic Oscillation (BAO) and strongly gravitationally lensed quasar-galaxy systems (combined data sets from SLACS and LSD surveys). These different tests falls into two distinct classes. The first one makes use of the angular diameter distance, and refers to the so called standard rulers. The second uses the luminosity distance and then we deal with standard candles. The two distance concepts, although theoretically related to each other, in practice have different systematic uncertainties and different parameter degeneracies. Hence their joint analysis is more restrictive in the parameter space.

We have considered $\Lambda$ CDM model, two Quintessence scenarios (with constant and variable equation of state), generalized Chaplygin gas and braneworld (Dvali, Gabadagdze, Porrati - DGP) model. The best fits we obtained for the model parameters in joint analysis turned out to prefer cases effectively equivalent to $\Lambda \mathrm{CDM}$ model. Our findings are in agreement with parallel studies performed by other authors on different sets of diagnostic probes.

We also tried to answer question: "Which model is the best?" with the aid of two informationtheoretic criteria: the Akaike Criterion (AIC) and Bayesian Information Criterion(BIC). It lead us to similar conclusion that the concordance model $\Lambda \mathrm{CDM}$ is clearly preferred in joint analysis. The quintessence (both having constant or time varying equation of state), and Chaplygin gas get considerably less support from the data while the brane world (DGP) scenario is practically ruled out.

25th Texas Symposium on Relativistic Astrophysics

December 6-10, 2010

Heidelberg, Germany 


\section{Introduction}

Modern cosmology relies on quite firm observational ground. Its strength lies in consistency across independent pieces of evidence (like e.g CMB anisotropies [9], the large-scale distribution of galaxies [7], the observed abundances of light elements, etc.) rather than in single one, crucial experiment. Concordance model with only few parameters fits most of the the current data quite well. Currently accelerating expansion of the Universe (inferred from the SNIa Hubble diagram [14]) is the most important open issue in modern cosmology and physics. Explanation of its origin may be attributed to either unknown exotic material component with negative pressure - so called dark energy, to infra red modification of gravity at cosmological scale or requires to relax the assumption of homogeneity of the Universe.

We present the joint analysis of several dark energy models using four different kind of probes coming from supernovae Ia data, CMBR shift parameter R, BAO and strong lensing systems. These different tests, called diagnostics for short, have better constraining power when taken together than one single probe. Presented results are extension of previous work [5], where we considered two test coming from SnIa and strong lensing systems. The method, concerning lenses, was proposed by Biesiada [3] and also discussed in details by Grillo et al. [8].

Currently there exists strong evidence that the Universe is spatially flat. Combined analysis of WMAP5, BAO and supernova data [9] gives $\Omega_{t o t}=1.0050_{-0.0061}^{+0.0060}$. Hence we will assume flat $(k=0)$ FRW model from now on.

\section{Diagnostic probes}

Strong gravitationally lensed systems create opportunity to test cosmological models of dark energy in a way alternative to Hubble diagrams (from SNIa or GRBs). In cosmological context the source is most often a quasar with a galaxy acting as the lens. Strong lensing reveals itself as multiple images of the source. Their separations depend on angular-diameter distances to the lens and to the source, which in turn are determined by background cosmology. This opens a possibility to constraining the cosmological model provided that we have good knowledge of the lens model. It turns out that in vast majority of cases lenses are type E/SO galaxies, which are found to be structurally homologous and well characterized by approximately SIS (Singular Isothermal Sphere) density profile. The formula for the Einstein radius in a SIS lens is following

$$
\theta_{E}=4 \pi \frac{\sigma_{S I S}^{2}}{c^{2}} \frac{D_{l s}}{D_{s}}
$$

where $D_{l s}$ denotes(angular-diameter) distance between lens and source while $D_{s}$ - between observer and source. Provided one has reliable knowledge about lensing system: the Einstein radius $\theta_{E}$ and lens velocity dispersion $\sigma_{S I S}$, one can use such well studied systems to test background cosmology. We used a combined sample of $n=20$ strong lensing systems from the SLACS [16] and LSD [15] surveys, with good spectroscopic measurements of stellar central dispersions $\sigma_{0}$, which is taken as a good estimator of $\sigma_{S I S}$ (for a detailed discussion see e.g. [8]). 
The central relation which splits into theoretical and observable counterparts reads:

$$
\mathscr{D}^{\text {th }}\left(z_{l}, z_{s} ; \mathbf{p}\right)=\frac{D_{s}(\mathbf{p})}{D_{l s}(\mathbf{p})}=\frac{\int_{0}^{z_{s}} \frac{d z^{\prime}}{h\left(z^{\prime} ; \mathbf{p}\right)}}{\int_{z_{l}}^{z_{s}} \frac{d z^{\prime}}{h\left(z^{\prime} ; \mathbf{p}\right)}}=\frac{4 \pi \sigma_{0}^{2}}{c^{2} \theta_{E}}=\mathscr{D}^{o b s}
$$

It is worth to notice that in the method used here models enter through a distance ratio which makes the inferences independent on the Hubble constant value. Our findings depend, however, on the reliability of lens modelling (e.g. SIS assumption).

Cosmological model parameters (coefficients in the equation of state) are estimated by minimizing the chi-square function:

$$
\chi^{2}(\mathbf{p})=\sum_{i} \frac{\left(\mathscr{D}_{i}^{o b s}-\mathscr{D}_{i}^{t h}(\mathbf{p})\right)^{2}}{\sigma_{\mathscr{D}, i}^{2}}
$$

where the sum is over the sample and $\sigma_{\mathscr{D}, i}^{2}$ denotes the variance of $\mathscr{D}^{\text {obs }}$. In calculating $\sigma_{\mathscr{D}}$ we assumed that only velocity dispersion errors contribute and the Einstein radii are determined accurately.

As a next probe we used the CMB shift parameter $\mathrm{R}$, which is a scaled distance to the last scattering surface (at redshift $\left.z_{l s s}=1090\right) R(\mathbf{p})=\sqrt{\Omega_{m}} \int_{0}^{z_{l s s}} \frac{d z}{h(z ; \mathbf{p})}$ [11], where $\Omega_{m}$ is the present day matter density, $h(z)$ is dimensionless expansion rate which depends on cosmological model (through parameters $(\mathbf{p})$ ). The most recent value from WMAP7 [10] is $R=1.725 \pm 0.018$. For comparison between theory and observations we will use the chi-square function:

$$
\chi_{C M B}^{2}(\mathbf{p})=\frac{[R(\mathbf{p})-1.725]^{2}}{0.018^{2}}
$$

i.e. just "one data point" for joint analysis.

We have also considered Baryon Acoustic Oscillations (BAO) distance measurements at redshift $z=0.35$ in the form of $A$ parameter (which is dimensional combination of so called dilatation scale) suitably expressed as: $A(\mathbf{p})=\frac{\sqrt{\Omega_{m}}}{0.35}\left[\frac{0.35}{h(0.35 ; \mathbf{p})}\left(\int_{0}^{0.35} \frac{d z}{h(z ; \mathbf{p})}\right)^{2}\right]^{1 / 3}$ Its updated value is $A(0.35)=0.493 \pm 0.017$ [13]. The corresponding chi-square function reads:

$$
\chi_{B A O}^{2}=\frac{[A(\mathbf{p})-0.469]^{2}}{0.017^{2}}
$$

Those three probes described above falls into category of standard rulers. Dealing with these tests we have to do with characteristic lengths like the size of sound horizon, the Einstein radius, the preferred distance between galaxies (which is in fact statistical standard ruler).

The fourth probe, supernovae Ia falls into different category, namely standard candles. We will use the data set of $n=557$ supernovae given in [2] known as Union2 compilation. This data set contains redshifts $z_{i}$ and distance moduli $\mu_{i}$ together with their errors $\sigma_{i}$. This leads to the chi-square function:

$$
\chi_{\text {SNIa }}^{2}=\sum_{i=1}^{N=557}\left[\frac{\mu^{o b s}\left(z_{i}\right)-\mu^{t h}\left(z_{i} ; \mathbf{p}\right)}{\sigma_{i}}\right]^{2}
$$

where $\mu$ denotes distance modulus: $\mu:=m-M=5 \log _{10}\left(D_{L}(z ; \mathbf{p})\right)+25$ and $D_{L}(z)$ luminosity distance. 
The probes described above were combined by calculating joint likelihoods:

$$
\mathscr{L}_{\text {tot }}=\mathscr{L}_{\text {rul }} \times \mathscr{L}_{\text {cand }}=\mathscr{L}_{C M B} \times \mathscr{L}_{\text {BAO }} \times \mathscr{L}_{\text {Lens }} \times \mathscr{L}_{\text {SNIa }}
$$

which is equivalent to the assessment of the total chi-square function. Because standard rulers and standard candles probe distance measures based on different concepts (angular diameter distance and luminosity distance), one step before making a full joint fit we performed fits based on rulers and candles separately.

\section{Results}

We have considered five cosmological scenarios widely discussed in current literature, namely $\Lambda C D M$ model, Quintessence with constant equation of state, Chevalier-Polarski-Linder model, Generalized Chaplygin gas models and brane-world scenario [6]. By virtue of the Einstein equations, the expansion rate $H=\frac{\dot{a}}{a}$ is determined by some set of parameters like present expansion rate $H_{0}$, present density of (pressureless) matter $\Omega_{m}$, radiation $\Omega_{r}$ or any other material component $\Omega_{x}$ (if considered) and the equation of state parameter $w$ (assuming hydrodynamical energy momentum tensor with $p=w \rho$ relation) depending on particular tested model. We will use a shorthand notation of $\mathbf{p}$ for such parameters. Their full specification is given in Table 1. Technically speaking, testing cosmological models means to determine parameters $\mathbf{p}$.

In flat $\Lambda C D M$ cosmology, $\Omega_{m}$ is the only free parameter. The result of our combined analysis, i.e. $\Omega_{m}=0.274 \pm 0.014$ (see Table 3) should be compared with independent measurements. The only method sensitive exclusively to matter density comes form studying peculiar velocities of galaxies. For egzample Mohayaee and Tully [12] applied orbit retracing methods to motions in the local supercluster and obtained $\Omega_{m}=0.22 \pm 0.02$, which is also consistent with our findings. In the class of quintessence models, the ESSENCE supernova survey team [17] pinned down the equation of state parameter to the range $w=-1.07 \pm 0.09$ (stat) \pm 0.12 (systematics) and $\Omega_{m}=0.274_{-0.020}^{+0.033}$ (stat $1 \sigma$ ). These results are in perfect agreement with our results shown in Tables 2 and 3. Concerning Chevalier - Polarski - Linder parametrization the joint constraint from WMAP+BAO $+\mathrm{H}_{0}+\mathrm{SN}$ provided by [10] gives the bound $w_{0}=-0.93 \pm 0.13, w_{a}=-0.41_{-0.71}^{+0.72}$. Our combined analysis gives support to the models with varying equation of state very close to the $\Lambda C D M$ model.

In the class of generalized Chaplygin gas models one can see that standard candles and standard rulers consistently support values of $\alpha$ close to zero (and $A \approx 1$ ). This is in agreement with previous, independent fits (e.g. [4] ) including the most recent ones [18]. We can say that our combined analysis constrains the generalized Chaplygin gas scenario to cases effectively equivalent to $\Lambda C D M$ model.

Cosmological models in brane-world scenarios have been widely discussed in the literature. Quite recent paper [19] presents one of the most comprehensive analysis of brane-world models by considering jointly the data from supernovae, gamma-ray bursts, BAO, CMB peaks, the look back times and growth functions for the large scale structure. Their results obtained by using the Markov Chain Monte Carlo simulation yield $\Omega_{m}=0.266_{-0.0304}^{+0.0298}$ which perfectly agrees with our results of joint analysis reported in Table 3 . 


\begin{tabular}{cc}
\hline Model & Cosmological expansion rate $H(z)$ (the Hubble function) \\
\hline$\Lambda$ CDM & $H^{2}(z)=H_{0}^{2}\left[\Omega_{m}(1+z)^{3}+\Omega_{\Lambda}\right]$ \\
Quintessence & $H^{2}(z)=H_{0}^{2}\left[\Omega_{m}(1+z)^{3}+\Omega_{Q}(1+z)^{3(1+w)}\right]$ \\
Chevalier-Polarski-Linder & $H^{2}(z)=H_{0}^{2}\left[\Omega_{m}(1+z)^{3}+\Omega_{Q}(1+z)^{3\left(1+w_{0}+w_{a}\right)} \exp \left(\frac{-3 w_{a} z}{1+z}\right)\right]$ \\
Chaplygin Gas & $H(z)^{2}=H_{0}^{2}\left[\Omega_{m}(1+z)^{3}+\Omega_{C h}\left(A_{0}+\left(1-A_{0}\right)(1+z)^{3(1+\alpha)}\right)^{\frac{1}{1+\alpha}}\right]$ \\
Braneworld & $H(z)^{2}=H_{0}^{2}\left[\left(\sqrt{\Omega_{m}(1+z)^{3}+\Omega_{r_{c}}}+\sqrt{\Omega_{r_{c}}}\right)^{2}\right]$ \\
\hline
\end{tabular}

Table 1: Expansion rates $H(z)$ in the models tested. The quantities $\Omega_{i}$ represent fractions of critical density currently contained in energy densities of respective components.

\begin{tabular}{ccccc}
\hline Cosmological model & Best fit rulers & $\chi^{2}$ rulers & Best fit candles & $\chi^{2}$ candles \\
\hline$\Lambda$ CDM & $\Omega_{m}=0.273 \pm 0.018$ & $\chi^{2}=63.961$ & $\Omega_{m}=0.275 \pm 0.020$ & $\chi^{2}=663.641$ \\
Quintessence & $\Omega_{m}=0.262 \pm 0.035$ & $\chi^{2}=63.829$ & $\Omega_{m}=0.299 \pm 0.075$ & $\chi^{2}=663.532$ \\
& $w=-1.066 \pm 0.188$ & & $w=-1.070 \pm 0.215$ & \\
Chevalier-Polarski-Linder & $\Omega_{m}=0.276 \pm 0.055$ & $\chi^{2}=63.707$ & $\Omega_{m}=0.228 \pm 0.156$ & $\chi^{2}=663.695$ \\
& $w_{0}=-0.824 \pm 0.704$ & & $w_{0}=-0.993 \pm 0.207$ & \\
& $w_{a}=-0.757 \pm 2.148$ & & $w_{a}=0.609 \pm 1.071$ & \\
Chaplygin Gas & $\Omega_{m}=0.273 \pm 0.018$ & $\chi^{2}=63.961$ & $\Omega_{m}=0.275 \pm 0.020$ & $\chi^{2}=663.641$ \\
& $A=1.000 \pm 0.001$ & & $A=0.999 \pm 0.004$ & \\
Braneworld & $\alpha=-0.040 \pm 2.260$ & & $\alpha=0.006 \pm 0.372$ & \\
& $\Omega_{m}=0.345 \pm 0.021$ & $\chi^{2}=72.697$ & $\Omega_{m}=0.177 \pm 0.015$ & $\chi^{2}=664.276$ \\
\hline
\end{tabular}

Table 2: Fits to different cosmological models from: a) combined standard rulers data (R+BAO+Lenses) second and third column, b)from Union2 sample $n=557$ SNIa - two last column.

\begin{tabular}{ccc}
\hline$\Lambda$ CDM & $\Omega_{m}=0.274 \pm 0.014$ & $\chi^{2}=727.610$ \\
Quintessence & $\Omega_{m}=0.274 \pm 0.014$ & $\chi^{2}=727.603$ \\
& $w=-1.004 \pm 0.048$ & \\
Chevalier-Polarski-Linder & $\Omega_{m}=0.274 \pm 0.014$ & $\chi^{2}=727.584$ \\
& $w_{0}=-0.989 \pm 0.124$ & \\
& $w_{a}=-0.082 \pm 0.621$ & \\
Chaplygin Gas & $\Omega_{m}=0.274 \pm 0.014$ & $\chi^{2}=727.610$ \\
& $A=1.0 \pm 0.004$ & \\
Braneworld & $\alpha=-0.112 \pm 1.282$ & \\
\hline
\end{tabular}

Table 3: Joint R+BAO+Lenses+Union2 fits to different cosmological models.

\section{Choose the best model}

Minimizing the chi-square function is good approach for finding best fit parameters for a certain model. It is however insufficient for deciding whether the model itself is the best one (i.e which one is the best supported by data at hand) because it does not take into account the relative structural complexity of the models. This sort of questions can be answered with model selection techniques. Here we have used two information-theoretic criteria: the Akaike Criterion (AIC) and 


\begin{tabular}{ccccc}
\hline Model & AIC & $\Delta_{i}$ & $w_{i}$ & Odds against \\
\hline$\Lambda \mathrm{CDM}$ & 729.610 & 0. & 0.609 & 1. \\
Quintessence & 731.603 & 1.993 & 0.225 & 3. \\
Chevalier-Polarski-Linder & 733.584 & 3.974 & 0.084 & 7. \\
Chaplygin & 733.610 & 4.00 & 0.082 & 7. \\
Braneworld & 779.676 & 50.066 & $8.210^{-12}$ & $7.10^{10}$ \\
\hline
\end{tabular}

Table 4: AIC Model Selection Results.

\begin{tabular}{ccccc}
\hline Model & BIC & $B I C \Delta_{i}$ & BIC $w_{i}$ & BIC Odds against \\
\hline$\Lambda \mathrm{CDM}$ & 733.97 & 0. & 0.957 & 1. \\
Quintessence & 740.322 & 6.353 & 0.040 & 24. \\
Chevalier-Polarski-Linder & 746.663 & 12.693 & 0.002 & 570. \\
Chaplygin & 746.689 & 12.719 & 0.002 & 578. \\
Braneworld & 784.036 & 50.066 & $1.310^{-11}$ & $7.10^{10}$ \\
\hline
\end{tabular}

Table 5: BIC Model Selection Results.

Bayesian Information Criterion (BIC) due to Schwarz.

Akaike criterion is based on Kullback-Leibler divergence $I(f, g)$ between two distributions $f(x)$ and $g(x)$, which says, roughly speaking, how much of the information is lost when $g$ is used to approximate $f$. As was shown by Akaike [1] the quantity, called the Akaike Information Criterion:

$$
A I C=-2 \ln (\mathscr{L}(\hat{\mathbf{p}} \mid \text { data }))+2 K
$$

is an approximately unbiased estimator of the K-L divergence between the model at hand $g(x \mid \mathbf{p})$ and an unknown true model $f(x)$ which generated the data. In our case $A I C=\chi^{2}(\hat{\mathbf{p}} \mid$ data $)+2 K$. Useful informations can be inferred from Akaike differences $\Delta_{i}:=A I C_{i}-A I C_{\min }$ calculated over the whole set of alternative candidate models $i=1, \ldots, N$ where by $A I C_{\min }$ we denoted $\min \left\{A I C_{i} ; i=1, \ldots, N\right\}$. Comparing several models, the one which minimizes AIC could be considered the best. Relative likelihoods of the models $\mathscr{L}\left(g_{i} \mid\right.$ data $)$ normalized to unity are called Akaike weights $w_{i}$ and give us the posterior probability of a model. The odds against given model with respect to the best one can be calculated as ratios of model pairs $\frac{w_{i}}{w_{j}}=\frac{\mathscr{L}\left(g_{i} \mid \text { data }\right)}{\mathscr{L}\left(g_{j} \mid \text { data }\right)}$.

A very similar criterion is the so called Bayesian Information Criterion (BIC):

$$
B I C=-2 \ln (\mathscr{L}(\hat{\mathbf{p}} \mid \text { data }))+K \ln (n)
$$

where $n$ is sample size and as previously $K$ denotes number of parameters. Its derivation stems from estimating the marginal likelihood of the data.

\section{Conclusions}

In this paper we performed joint analysis of five cosmological models invoked to explain accelerating expansion of the Universe. We used the data from strong gravitational lensing systems, CMB acoustic peak location and BAO data in combination with supernovae Ia data (Union2 compilation). The probes we used came both from standard rulers and standard candles. They 
invoke different (although theoretically related) concepts of a distance in cosmology, hence they have different parameter degeneracies and different restrictive power in the parameter spaces of cosmological models.

We used two information-theoretic methods to assess which model is the best supported by data, namely AIC and BIC. Our main conclusion (see tables 4 and 5) is that the concordance model $\Lambda \mathrm{CDM}$ is the preferred one and brane world scenario is practically irrelevant - both criteria give the same result. The first criterion Akaike implies that the support given by the data to Quintessential model even though less is comparable to the concordance model. Both models with a dynamical equation of state $w(z)$ Chevalier-Polarski-Linder (CPL parametrization) and Chaplygin gas scenarios get considerably less (and similar to each other) support from the data, but with the evidence against them assessed as moderate. According to the second criterion BIC we get much stronger evidence against Quintessence with constant equation of state. The judgement of this criterion is even stronger against models with dynamical equation of state, so that they are practically ruled out. Odds against the brane-world scenario are so high that finally this scenario (DGP model) should be abandoned according to both criteria.

Acknowledgments: This work was supported by the Polish Ministry of Science Grant no. N N203 390034.

\section{References}

[1] H. Akaike, IEEE Transactions on Automatic Control 19 (1974) 716.

[2] R. Amanullah et al., Astrophys.J. 716 (2010) 712.

[3] M. Biesiada, Phys. Rev. D 73 (2006) 023006.

[4] M. Biesiada,W. Godłowski and M. Szydłowski, Astrophys. J. 622 (2005) 28.

[5] M. Biesiada, A. Piórkowska and B. Malec, Mon. Not. R. Astron. Soc. 406 (2010) 1055.

[6] G. Dvali, G. Gabadadze and M. Porrati, Phys. Lett. B 485 (2010) 208.

[7] D.J. Eisenstein and W. Hu, Astrophys.J. 511 (1999) 5.

[8] C. Grillo, M. Lombardi and G. Bertin, Astron. Astrophys. 477 (2008) 397.

[9] G. Hinshaw et al., Astrophys.J. Suppl. 180 (2009) 225.

[10] E. Komatsu et al., Astrophys.J. Suppl. 180 (2009) 330.

[11] L. Page et al., Astrophys.J. Suppl. 148 (2003) 233.

[12] R. Mohayaee and B. Tully, Astrophys. J. 635 (2005) L113.

[13] B. Reid et al., Mon.Not.Roy.Astron.Soc. 404 (2010) 60.

[14] A.G. Riess et al., Astron. J. 116 (1998) 1009.

[15] T. Treu and L.V.E. Koopmans, Astrophys.J.611 (2004) 739.

[16] T. Treu et al., Astrophys.J.650 (2006) 1219.

[17] W.M. Wood-Vasey et al., Astrophys.J. 666 (2007) 694.

[18] P. Wu and H. Yu, Astrophys. J. 658 (2007) 663.

[19] L. Xu and Y. Wang, Phys Rev. D 82 (2010) 043503. 